\title{
Eigenstructure Based Partially Adaptive Array Design
}

\author{
BARRY D. VAN VEEN, MEMBER, IEEE
}

\begin{abstract}
A procedure is presented for designing partially adaptive arrays having nearly fully adaptive performance under steady-state conditions. Theory predicts that the required adaptive dimension is less than or equal to the rank of the spatially/temporally correlated portion of the interference correlation matrix for arbitrary linearly constrained minimum variance beamformers. Knowledge of the eigenstructure of the interference correlation matrix is required to implement a beamformer with this adaptive dimension. To avoid adaptive estimation of the eigenstructure, the eigenstructure of an "averaged" correlation matrix, which spans the interference scenarios of interest, is utilized, and the adaptive dimension is given by the rank of the averaged correlation matrix. Simulations illustrate the effectiveness of this approach.
\end{abstract}

\section{INTRODUCTION}

$\mathrm{B}$ EAMFORMERS using a portion of the available adaptive degrees of freedom are known as partially adaptive arrays [1], [2]. It is desireable to minimize the adaptive degrees of freedom in a beamformer since adaptive algorithms represent a significant computational expense and directly affect overall system cost. In addition, reducing adaptive dimension can result in faster adaptive response [2], [3]. However, cancellation performance usually degrades also. Minimizing performance degradation is a primary consideration in partially adaptive array design.

A variety of approaches have been suggested for designing partially adaptive arrays [1]-[5]. The method of Owsley [5] is particularly relevant to the discussion which follows. In [5] it is shown that for a narrow-band signal environment and a unity gain constraint in the desired signal direction, one can obtain fully adaptive performance using a number of adaptive weights equal to the rank of the spatially correlated portion of the interference correlation matrix. However, one must have knowledge of the interference correlation matrix eigenstructure to implement this scheme.

Here a similar result is derived for narrow-band or broadband signals with arbitrary linear constraints on the array response. The minimal adaptive dimension required for fully adaptive performance is shown to be equal to the rank of the spatially/temporally correlated portion of the interference correlation matrix. As in [5], implementation utilizes a transformation which spans the eigenstructure of the interference correlation matrix. However, in this approach the

Manuscript received April 1, 1987; revised August 14, 1987. This work was supported in part by the Army Research Office, Electronics Division, under Contract DAAG29-84-K-0014.

The author is with the Department of Electrical and Computer Engineering, University of Wisconsin-Madison, 1415 Johnson Drive, Madison, WI 53706-1691.

IEEE Log Number 8718772 requirement of estimating the interference eigenstructure is circumvented by choosing a transformation which spans the eigenstructure for all interference scenarios of interest. The resulting adaptive dimension is dependent on the array geometry and interference scenarios of interest.

Section II shows that the adaptive weight vector in the generalized sidelobe canceller (GSC) lies in a subspace with dimension given by the rank of the spatially/temporally correlated portion of the interference correlation matrix. Construction of a transformation for reducing adaptive dimension is addressed in Section III. Simulations illustrating the utility of the eigenstructure approach are given in Section IV followed by a summary in Section V.

\section{Adaptive Dimension in the GSC}

The output of a beamformer $y(k)$ is expressed here as the inner product of a weight vector $W$ and the total collection of data in the array, $X(k): y(k)=W^{T} X(k) . W$ and $X(k)$ are assumed to be $N$-dimensional vectors. The linearly constrained minimum variance beamforming (LCMVB) problem for selecting the weights is

$$
\min _{W} W^{T} R_{x} W \text { subject to } C^{T} W=F
$$

where $R_{x}=E\left\{X(k) X^{T}(k)\right\}$ is the $N$ by $N$ correlation matrix of the data, $C$ is the $N$ by $L$ constraint matrix, and $F$ is the $L$ dimensional response vector.

The GSC has been suggested as an alternative implementation to the Frost beamformer [12] and has a number of advantages for LCMVB [6]. A block diagram of the GSC is given in Fig. 1. The weight vector of (1) is decomposed into two terms: $W=W_{0}-C_{n} W_{n}$. $W_{0}$ is a fixed beamformer satisfying $C^{T} W_{0}=F, C_{n}$ is an $N$ by $M$ rank $M$ signal blocking matrix satisfying $C^{T} C_{n}=0$, and $W_{n}$ is an $M$ dimensional unconstrained adaptive weight vector. If $M=N$ - $L$, then the GSC and Frost beamformer have equivalent steady-state performance. If $C_{n}$ is chosen to satisfy $C_{n}^{T} W_{0}=0$ in addition to $C_{n}^{T} C=0$, then $W_{0}$ determines the quiescent response [7].

The weight vector $W_{n}$ is obtained as the solution to the unconstrained minimization problem

$$
\min _{W_{n}}\left(W_{0}-C_{n} W_{n}\right)^{T} R_{x}\left(W_{0}-C_{n} W_{n}\right)
$$

which is given by

$$
W_{n}=\left(C_{n}^{T} R_{x} C_{n}\right)^{-1} C_{n}^{T} R_{x} W_{0}
$$




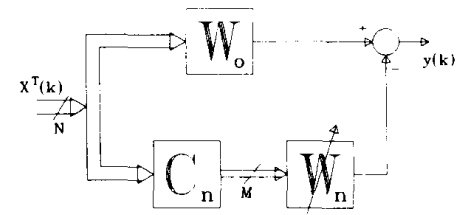

Fig. 1. Generalized sidelobe canceller (GSC).

Assume that $R_{x}$ can be decomposed into two terms: $R_{s}$, corresponding to the signal of interest, and $R_{I}$, corresponding to interference and noise; $R_{x}=R_{s}+R_{l}$. If the signal blocking matrix is designed properly, then it must be orthogonal to the source correlation matrix, or $C_{n}^{T} R_{s}=0$, and $W_{n}$ depends only on $R_{I}$

$$
W_{n}=\left(C_{n}^{T} R_{l} C_{n}\right)^{-1} C_{n}^{T} R_{I} W_{0} .
$$

Represent $R_{I}$ as the sum of a rank $K$ spatially/temporally correlated term and an uncorrelated term corresponding to additive white noise

$$
R_{I}=E S E^{T}+\sigma^{2} I
$$

where $E$ is an $N$ by $K$ matrix of eigenvectors and $S$ is a diagonal matrix containing the $K$ eigenvalues of the correlated portion of $R_{l}$. Substituting (5) into (4) results in an expression for $W_{n}$ in terms of the eigenvectors $E$.

Beginning with the term in the inverse we have

$$
C_{n}^{T} R_{I} C_{n}=\tilde{E} S \tilde{E}^{T}+\sigma^{2} I
$$

where $\tilde{E}$ is $C_{n}^{T} E$ and we have assumed $C_{n}^{T} C_{n}=I$. This does not cause a loss of generality since it is the space which $C_{n}$ spans that is of importance, not the value of its elements. The inverse of (6) is obtained using the modified matrices formula [8] as

$$
\left(C_{n}^{T} R_{I} C_{n}\right)^{-1}=\sigma^{-2} I-\sigma^{-4} \tilde{E}\left(\sigma^{-2} \tilde{E}^{T} \tilde{E}+S^{-1}\right)^{-1} \tilde{E}^{T} .
$$

$C_{n}^{T} R_{J} W_{0}$ becomes

$$
\begin{aligned}
C_{n}^{T} R_{I} W_{0} & =\tilde{E} G+\sigma^{2} C_{n}^{T} W_{0} \\
& =\tilde{E} G
\end{aligned}
$$

where $G=S E^{T} W_{0}$, and it is assumed that $C_{n}$ is designed so $W_{0}$ provides the quiescent response of the beamformer [7], thus $C_{n}^{T} W_{0}=0$. Combining (7) and (8) yields

$$
W_{n}=C_{n}^{T} E H
$$

with the $K$-dimensional vector $H$ as

$$
H=\left[\sigma^{-2} I-\sigma^{-4}\left(\sigma^{-2} \tilde{E}^{T} \tilde{E}+S^{-1}\right)^{-1} \tilde{E}^{T} \tilde{E}\right] G .
$$

Equation (9) gives the desired result. The adaptive weight vector lies within a subspace which is at most $K$-dimensional, namely, the space spanned by $C_{n}^{T} E$. Thus assuming $E$ is known, one can reduce the adaptive dimensionality of the system from $M$ to $K$ with no loss in cancellation performance. However, $E$ is not generally known a priori and computing $E$ adaptively would represent a significant computational expense.

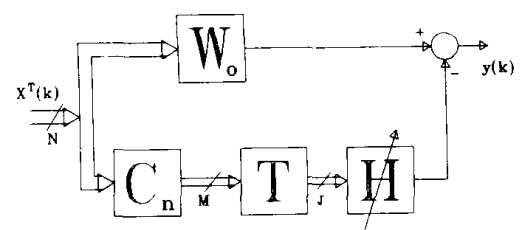

Fig. 2. Partially adaptive generalized sidelobe canceller.

\section{Designing the Partially Adaptive GSC}

Assuming for the moment that $E$ is known, the GSC is modified as indicated in Fig. 2 to reduce adaptive dimensionality with no loss in performance. $T$ is an $M$ by $K(J=K)$ dimensional transformation whose columns are a basis for the space spanned by the columns of $C_{n}^{T} E$. If $E$ is not known, then the problem is to find a $T$ of smallest $J(J \geqq K)$ that spans the space in which $W_{n}$ lies for all interference scenarios of interest.

Define $R(\theta)$ to be the correlation matrix for an interferer parameterized by a vector $\theta$. For a simple case $\theta$ could represent the direction of the interferer with respect to the array. More generally, $\theta$ could represent direction, temporal frequency characteristics, spatial distribution, and uncertainties in sensor position. Define an "averaged" correlation matrix $\hat{R}$ as

$$
\hat{R}=\int_{\theta_{a}}^{\theta_{b}} R(\theta) d \theta .
$$

The space spanned by $\hat{R}$ includes the space spanned any $R(\theta)$ for $\theta \in\left[\theta_{a}, \theta_{b}\right]$. For example, let $\theta$ represent the direction of a spatial point source at the array and assume its temporal frequency characteristics are known. In this case $R(\theta)$ is the correlation matrix of the source located at $\theta$ and $\hat{R}$ is an "average" of correlation matrices for sources arriving between directions $\theta_{a}$ and $\theta_{b}$. Any source with direction $\theta_{a} \leqq \theta$ $\leqq \theta_{b}$ lies in the space spanned by $\hat{R}$. Note that in general $\left[\theta_{a}, \theta_{b}\right]$ may represent a union of several disjoint regions.

Assuming enough a priori knowledge of the interference environment is available to construct a representative $\hat{R}$, the transformation $T$ is chosen as a basis for the space spanned by $C_{n}^{T} \hat{R} C_{n}$. This is easily accomplished by composing $T$ of the eigenvectors of $C_{n}^{T} \hat{R} C_{n}$ corresponding to nonzero eigenvalues. The resulting partially adaptive GSC has fully adaptive performance for any interference environment contained within the parameterization region since $T$ spans the space in which $C_{n}^{T} E$ lies for any $\theta \in\left[\theta_{a}, \theta_{b}\right]$.

The rank of $C_{n}^{T} \hat{R} C_{n}$ is important since it determines the number of adaptive weights required. In general, it depends on the array geometry, $C_{n}$, and the parameterization region $\left[\theta_{a}, \theta_{b}\right]$ and thus must be evaluated numerically for the specific case of interest. However, some insight into the behavior of the rank of $\hat{R}$ can be obtained using the results of Buckley [9].

In [9] it is shown that the rank of the correlation matrix for a broad-band source of bandwidth $B$ arriving at the array from angle $\phi$ is approximately given by

$$
\lceil B T(\phi) / \pi+1\rceil
$$

where $T(\phi)$ represents the total temporal aperture of the array 
presented to a source at $\phi$ and $\lceil x\rceil$ indicates the next integer greater than $x$. Equation (11) indicates that the rank of an interferer increases as the bandwidth and/or the temporal aperture increases. Therefore, the rank of $\hat{R}$ (and similarly the rank of $C_{n}^{T} \hat{R} C_{n}$ ) is expected to increase with increasing bandwidth and/or temporal aperture.

Numerically determining the rank of a matrix is a difficult problem. Following [10] one approach is to choose the rank as the value $J$ for which

$$
\frac{\sum_{i=1}^{J} \lambda_{i}}{\sum_{i=1}^{M} \lambda_{i}}>\alpha
$$

where the $\lambda_{i}$ are the ordered eigenvalues $\lambda_{1} \geqq \lambda_{2} \geqq \cdots \geqq \lambda_{M}$ of $C_{n}^{T} \hat{R} C_{n}$. The constant $\alpha$ is chosen less than or equal to one. $(1-\alpha)^{*} 100$ determines the percentage error incurred by representing the space spanned by $C_{n}^{T} \hat{R} C_{n}$ with $J$ eigenvectors.

Numerical eigenvalue determination will not yield exactly zero eigenvalues, so choosing $\alpha$ involves a compromise. Selecting $\alpha$ too small results in poor performance, while selecting $\alpha$ too large increases the adaptive dimension unnecessarily. Simulations have indicated that $\alpha$ must be increased as the severity of the interference threat increases to maintain nearly fully adaptive performance. For example, a value of 0.999 may be sufficient for nulling $40-\mathrm{dB}$ (relative to white noise) interferers, but a value of 0.9999 may be required to achieve fully adaptive performance with $60-\mathrm{dB}$ interferers. In the simulations presented in the following section, a value of 0.999 is satisfactory for interferers having a 20-percent relative bandwidth, but a value of 0.9999 is required for a 40 percent relative bandwidth. Buckley [9] makes a similar observation concerning the number of eigenvector constraints required to null broad-band sources. Detailed investigation of the minimum $\alpha$ required as a function of bandwidth and power is beyond the scope of the present work.

\section{Simulations}

Simulations are presented in this section to verify the effectiveness of the partially adaptive design technique discussed above and illustrate the potential for reducing the number of adaptive weights in a broad-band beamformer for several interference scenarios. In the cases considered $\theta$ represents the interferer direction of arrival at the array relative to broadside. The interferers are assumed to be real and white on a fixed temporal bandwidth, and the integral of (10) is approximated with a sum.

The array configuration considered is an eight-element linear equal spaced array having 12 tap FIR filters in each sensor channel. The sensors are spaced at one-half wavelength for the highest frequency component, and the tap delay is normalized to one second, resulting in a normalized temporal frequency range of $[-\pi, \pi]$. The constraint matrix is designed to pass signals from broadside on the frequency range of interest using the approach of [9]. The quiescent response is designed using the least squares method of [9] to have unit gain in the broadside direction and zero gain at angles greater than $0.1 \mathrm{rad}$ from broadside on the frequency range of interest. Given the constraint matrix $(C)$ and quiescent response $\left(W_{0}\right)$, the signal blocking matrix $\left(C_{n}\right)$ is computed as the null space of the matrix $\left[C, W_{0}\right]$.

Two different interference bandwidths are considered. The first case has frequency range $[0.6 \pi, 0.8 \pi]$ (20-percent relative bandwidth), and the second has frequency range $[0.4 \pi, 0.8 \pi]$ (40-percent relative bandwidth). Fig. 3 illustrates the broad-band quiescent beam patterns for the 20-percent relative bandwidth system. A similar pattern results for the 40 percent relative bandwidth system.

The partially adaptive array performance is evaluated by comparing fully and partially adaptive array gains for an interference environment consisting of two interferers in additive white noise. The interference to white noise levels are 30 and $40 \mathrm{~dB}$. The gain is evaluated on a grid of 102 points: 17 directions on $[-\pi / 2, \pi / 2]$ (30-dB interferer) for each of six directions on $[0.15, \pi / 2]$ (40-dB interferer). Tables I and II summarize the performance of several different partially adaptive designs for 20 - and 40 -percent relative bandwidths, respectively.

In each table four different design examples are examined corresponding to various a priori assumptions on the directions of interferers. For example, design 3 corresponds to the case where one is concerned only with interferers located at positive angles relative to broadside. The average and maximum degradation on the design region is computed using the difference between fully and partially adaptive array gains at the grid points where both interferers are located within the design region. The maximum degradation outside the design region is computed similarly, using grid points where either interferer is outside the design region. The adaptive dimension of the fully adaptive arrays implementing the desired constraints and quiescent response are 87 and 85 for Tables I and II respectively. Typical fully adaptive array gains are $50 \mathrm{~dB}$ for the 20-percent bandwidth system and $40-50 \mathrm{~dB}$ for the 40 percent bandwidth system.

Detailed examination of performance indicates that the maximum degradation on the design region occurs at isolated points. This is supported by the low values of average degradation. For example, design 1 in Table I has a maximum degradation of $5.71 \mathrm{~dB}$, but the degradation is less than $0.5 \mathrm{~dB}$ at 96 of the 102 grid points. The maximum occurs when the $30-$ and $40-\mathrm{dB}$ interferers arrive at -0.12 and $0.15 \mathrm{rad}$, respectively, requiring two nulls very close to broadside. Performance is improved with a larger value of $\alpha$ at the expense of additional adaptive weights. Performance outside the design region is worse, as expected, but remains within 10 $\mathrm{dB}$ of fully adaptive for the cases considered.

Typical performance for the partially adaptive designs is illustrated in more detail in Figs. 4-6. The response of design 3 from Table I ( 28 adaptive weights) is compared to that of a fully adaptive system for an environment consisting of two broad-band interferers in white noise. The $30-$ and $40-\mathrm{dB}$ (relative to white noise) interferers are located at direction sines of 0.38 and 0.66 , respectively. Fig. 4 depicts the fully (dashed) and partially (solid) adaptive broad-band beam 


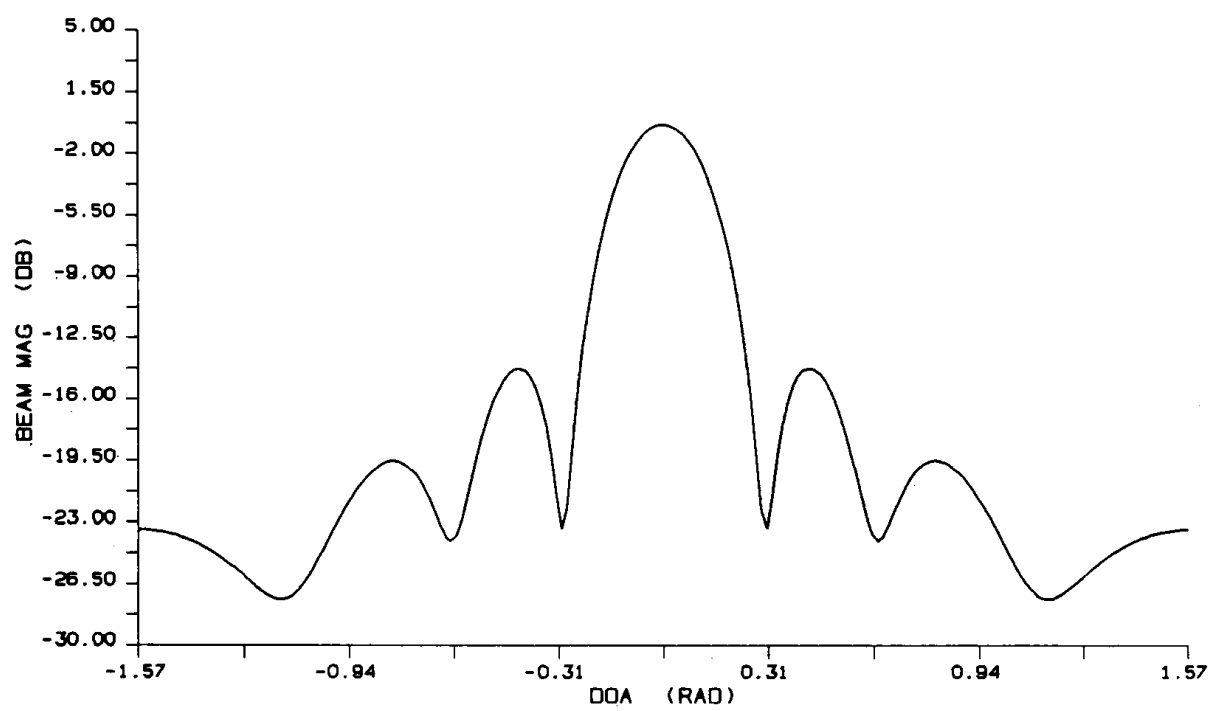

Fig. 3. Broad-band quiescent beam pattern for eight-element linear equal spaced array with 12 tap FIR filters per channel. Design bandwidth $[0.6 \pi, 0.8 \pi]$.

TABLE I

PARTIALLY ADAPTIVE PERFORMANCE SUMMARY FOR DESIGN BANDWIDTH $[0.6 \pi, 0.8 \pi], \alpha=0.999^{\mathrm{a}}$

\begin{tabular}{ccccccc}
\hline & $\begin{array}{c}\text { Design } \\
\text { Number }\end{array}$ & $\begin{array}{c}\text { Regign } \\
{\left[\theta_{a}, \theta_{b}\right]}\end{array}$ & $\begin{array}{c}\text { Adaptive } \\
\text { Dimension }\end{array}$ & $\begin{array}{c}\text { Average Gain } \\
\text { Degradation } \\
\text { on Region }\end{array}$ & $\begin{array}{c}\text { Maximum Gain } \\
\text { Degradation } \\
\text { on Region }\end{array}$ & $\begin{array}{c}\text { Maximum Gain } \\
\text { Degradation } \\
\text { Outside Region }\end{array}$ \\
\hline 1 & -1.57 & -0.1 & & & & \\
2 & 0.1 & 1.57 & 40 & 0.27 & 5.71 & N/A \\
3 & -0.785 & -0.2 & 34 & 0.21 & 2.54 & 5.71 \\
4 & 0.2 & 0.785 & 28 & 0.27 & 2.42 & 7.39 \\
& 0.1 & 1.57 & 28 & 0.47 & 2.85 & 8.03 \\
\hline
\end{tabular}

a Fully adaptive dimension is 87 . Angles in rad, gain in $\mathrm{dB}$.

TABLE II

PARTIALLY ADAPTIVE PERFORMANCE SUMMARY FOR DESIGN BANDWIDTH $[0.4 \pi, 0.8 \pi], \alpha=0.9999^{\text {a }}$

\begin{tabular}{|c|c|c|c|c|c|c|}
\hline \multirow{2}{*}{$\begin{array}{c}\begin{array}{c}\text { Design } \\
\text { Number }\end{array} \\
1\end{array}$} & \multicolumn{2}{|c|}{$\begin{array}{l}\text { Design } \\
\text { Region } \\
{\left[\theta_{a}, \theta_{b}\right]}\end{array}$} & \multirow{2}{*}{$\begin{array}{c}\begin{array}{c}\text { Adaptive } \\
\text { Dimension }\end{array} \\
63\end{array}$} & \multirow{2}{*}{$\begin{array}{c}\begin{array}{c}\text { Average Gain } \\
\text { Degradation } \\
\text { on Region }\end{array} \\
0.22\end{array}$} & \multirow{2}{*}{$\begin{array}{c}\begin{array}{c}\text { Maximum Gain } \\
\text { Degradation } \\
\text { on Region }\end{array} \\
1.74\end{array}$} & \multirow{2}{*}{$\begin{array}{c}\begin{array}{c}\text { Maximum Gain } \\
\text { Degradation } \\
\text { Outside Region }\end{array} \\
\text { N/A }\end{array}$} \\
\hline & $\begin{array}{c}-1.57 \\
0.1\end{array}$ & $\begin{array}{c}-0.1 \\
1.57\end{array}$ & & & & \\
\hline 2 & $\begin{array}{c}-0.785 \\
0.2\end{array}$ & $\begin{array}{l}-0.2 \\
0.785\end{array}$ & 55 & 0.17 & 0.49 & 1.78 \\
\hline 3 & 0.1 & 1.57 & 46 & 0.41 & 1.79 & 4.20 \\
\hline 4 & 0.1 & 0.785 & 37 & 0.51 & 1.63 & 4.62 \\
\hline
\end{tabular}

a Fully adaptive dimension is 85 . Angles in rad, gain in $\mathrm{dB}$. 


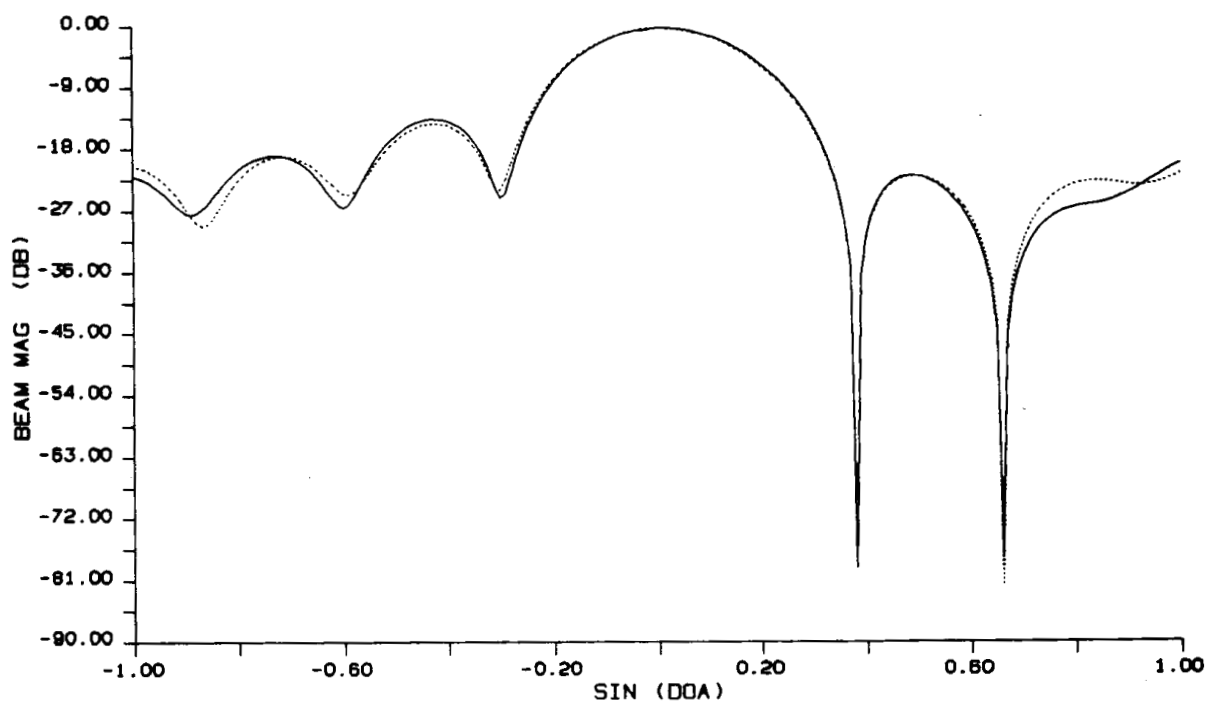

Fig. 4. Broad-band beam patterns when two interferers arrive at direction sines of 0.38 and 0.66 . Design bandwidth $[0.6 \pi, 0.8 \pi]$. Partially adaptive beam pattern (solid line) obtained using design 3 from Table I (28 adaptive weights). Dashed line represents fully adaptive beam pattern.

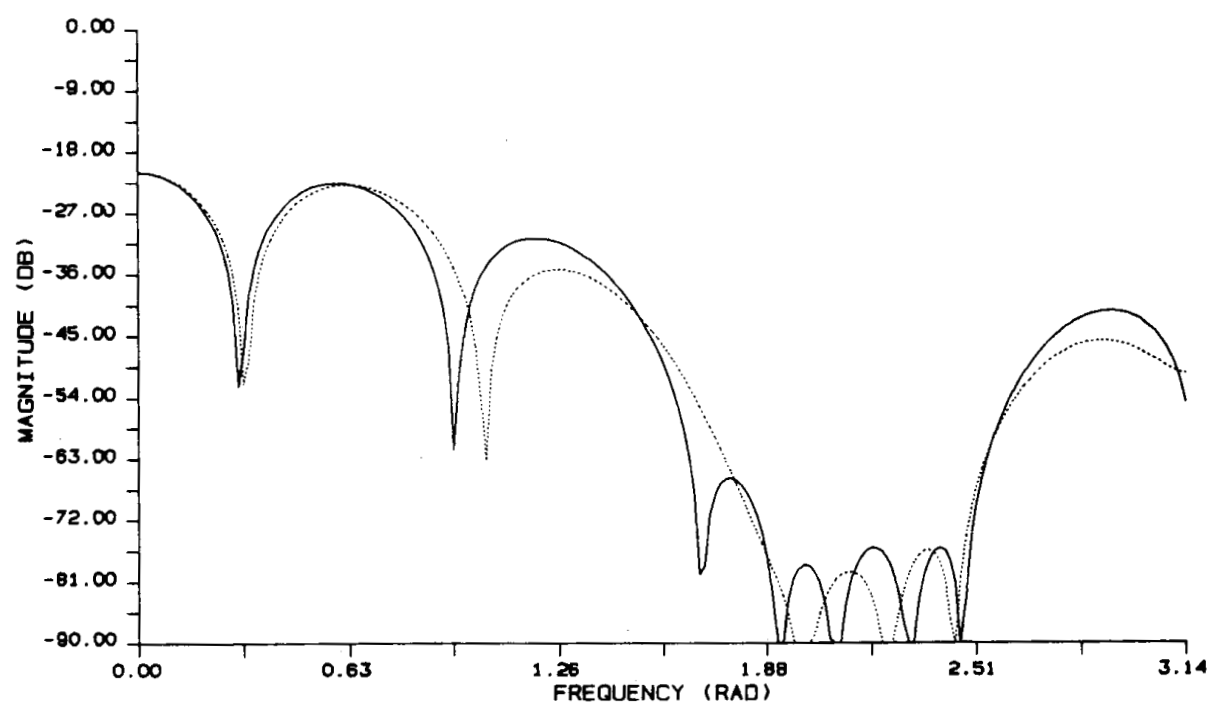

Fig. 5. Frequency response magnitude for systems of Fig. 4 at direction sine of 0.38 . Interferer extends from $1.885(0.6 \pi)$ to 2.513 $(0.8 \pi)$ radians. Solid line represents partially adaptive, dashed line represents fully adaptive. 


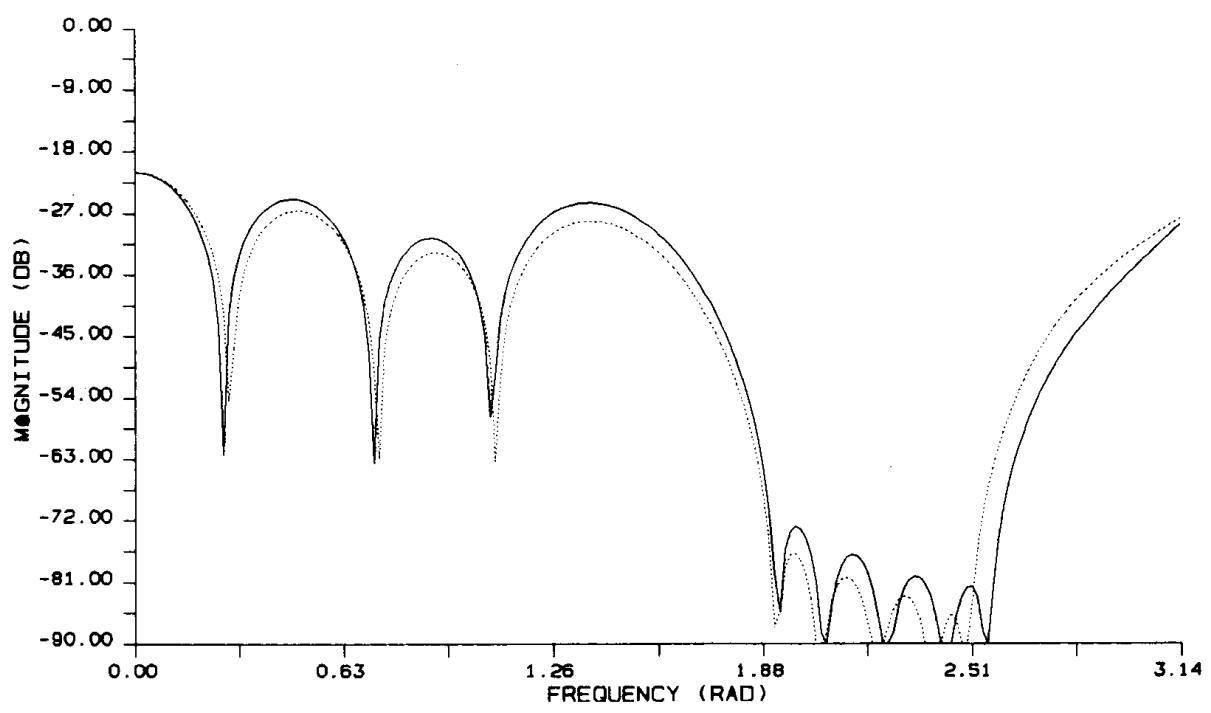

Fig. 6. Frequency response magnitude for systems of Fig. 4 at direction sine of 0.66 . Interferer extends from $1.885(0.6 \pi)$ to 2.513

$(0.8 \pi)$ radians. Solid line represents partially adaptive, dashed line represents fully adaptive.

patterns. The beam patterns are almost identical, although the fully adaptive system null is about $3 \mathrm{~dB}$ deeper at 0.66 . The frequency response magnitude in the interferer directions is shown in Figs. 5 and 6. The interferer bandwidth is from $1.885(0.6 \pi)$ to $2.513(0.8 \pi)$. The solid and dashed lines represent partially and fully adaptive response. The interference rejection of the partially adaptive design is nearly equivalent to that of fully adaptive across the frequency band.

The adaptive dimension decreases with bandwidth and size of the design region as indicated by (11). Note that the 20percent bandwidth examples require only one-fourth to onehalf the adaptive weights of the fully adaptive system while the 40 -percent bandwidth examples require one-half to threefourths of the number of fully adaptive weights. This can result in substantial complexity reduction, depending on the specific adaptive algorithm implemented. For example, with the sample matrix inversion algorithm [11] reducing adaptive dimension by one-half reduces the complexity of estimating the sample covariance matrix by a factor of four and the matrix inversion by a factor of eight.

\section{SUMMARY}

A procedure is presented for designing partially adaptive arrays based on the eigenstructure of an averaged interference correlation matrix. Theoretical results predict that this approach results in partially adaptive arrays having steady-state performance equivalent to fully adaptive and the number of adaptive weights required is given by the rank of the averaged interference correlation matrix. Simulations illustrate the effectiveness of the technique.

\section{ACKNOWLEDGMENT}

I would like to thank Professor Richard Roberts for his support, helpful comments, and numerous stimulating discussions on partially adaptive arrays.

\section{REFERENCES}

[1] D. R. Morgan, "Partially adaptive array techniques," IEEE Trans. Antennas Propagat., vol. AP-26, pp. 823-833, Nov. 1978.

[2] D. J. Chapman, "Partial adaptivity for the large array," IEEE Trans. Antennas Propagat., vol. AP-24, pp. 685-696, Sept. 1976.

[3] W. F. Gabriel, "Using spectral estimation techniques in adaptive processing antenna systems," IEEE Trans. Antennas Propagat., vol. AP-34, pp. 291-300, Mar. 1986.

[4] B. D. Van Veen and R. A. Roberts, "Partially adaptive beamformer design via output power minimization," IEEE Trans. Acoust. Speech, Signal Proc., vol. ASSP-35, pp. 1524-1532, Nov. 1987.

[5] N. L. Owsley, in Array Signal Processing, S. Haykin, Ed. Englewood Cliffs, NJ: Prentice-Hall, 1985.

[6] L. J. Griffiths and C. W. Jim, "An alternative approach to linearly constrained adaptive beamforming," IEEE Trans. Antennas Propagat., vol. AP-30, pp. 27-34, Jan. 1982.

[7] L. J. Griffiths and K. M. Buckley, "Quiescent pattern control in linearly constained adaptive arrays," IEEE Trans. Acoust. Speech, Signal Proc., vol. ASSP-35, pp. 917-926, July 1987.

[8] T. Kailath, Linear Systems. Englewood Cliffs, NJ: Prentice-Hall, 1980.

[9] K. M. Buckley, "Spatial/spectral filtering with linearly constrained minimum variance beamformers," IEEE Trans. Acoust. Speech, Signal Proc., vol. ASSP-35, pp. 249-266, Mar. 1987.

[10] M. H. Er and A. Cantoni "A new set of linear constraints for broadband time domain element space processors," in ICASSP 85 Proc., pp. 46.7.1-46.7.4, Apr. 1985.

[11] I. S. Reed, J. D. Mallet, and L. E. Brennan, "Rapid convergence rate in adaptive arrays," IEEE Trans. Aerosp. Electron. Syst., vol. AES 10. pp. 853-863, Nov. 1974.

[12] O. L. Frost, "An algorithm for linearly constrained adaptive array processing," Proc. IEEE, vol. 60, pp. 926-935, Aug. 1972.

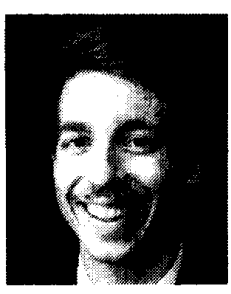

Barry D. Van Veen (S'81-S'83-M'83-M'86) was born in Green Bay, Wl, on June 20,1961 . He received the B.S. degree from Michigan Technological University, Houghton, in 1983 and the Ph.D. degree from the University of Colorado, Boulder, in 1986 , both in electrical engineering.

In the spring of 1987 he was with the Department of Electrical and Computer Engineering at the University of Colorado-Boulder. Since August of 1987 he has been with the Department of Electrical and Computer Engineering at the University of Wisconsin-Madison as an Assistant Professor. His research interests include array processing, spectral estimation, and adaptive algorithms.

Dr. Van Veen was an ONR Fellow while working on the Ph.D. degree. 